\title{
Bone SPECT/CT imaging of a liposclerosing myxofibroid tumor in an unexpected localization
}

Willm Uwe Kampen ${ }^{1}$, Lidija Antunovic ${ }^{2^{*}}$ (D) Andreas M. Luebke ${ }^{3}$, Guido Sauter ${ }^{3}$, Klaus Strobel ${ }^{4}$ and Frederic Paycha ${ }^{5}$

\author{
* Correspondence: lidija.antunovic@ \\ humanitas.it \\ ${ }^{2}$ Department of Nuclear Medicine, \\ Humanitas Clinical and Research \\ Center, Rozzano, Milan, Italy \\ Full list of author information is \\ available at the end of the article
}

\begin{abstract}
Background: The combination of both functional and morphological information using three-phase bone scintigraphy and tomographic imaging with single photon emission tomography/computed tomography (SPECT/CT) allows precise characterization of most bone lesions. The hybrid imaging is most useful when conventional imaging is equivocal or contraindicated (e.g magnetic resonance imaging (MRI).
\end{abstract}

Case presentation: A 69-year old, male patient with moderate pain in his posterior pelvic region was diagnosed with an unclear tumor of the iliac bone. Conventional radiographs, computed tomography (CT), and MRI imaging were inconclusive to confirm or refute a malignant process. There was no abnormal hyperperfusion on the early images. On the delayed images, moderately increased osteoblastic activity was noted, and the provisional diagnosis was in favor of a benign process.

Histopathology confirmed a benign liposclerosing myxofibrous tumor located in the left iliac bone.

Conclusion: Bone scan SPECT/CT might (a) help in classifying bone lesions by combining their metabolic and morphological (e.g CT) characteristics and (b), might help in discriminating benign and malignant tumors.

Keywords: Liposclerosing myxofibrous tumor (LSMFT), Fibrous dysplasia (FD), Bone scintigraphy, SPECT/CT

\section{Background}

A benign, fibro-osseous lesion consisting of a mixture of different tissues with lipomatous, myxofibromatous or fibroxanthomatous components, with occasional cyst formation, necrotic areas or ischemic ossification was first described and labeled as a liposclerosing myxofibrous tumor (LSMFT) by Ragsdale and Sweet in 1986 (Ragsdale et al., 1986). This tumor has a strong predilection for the intertrochanteric region of the femur (80-90\%) and a risk of malignant transformation in approximately $10 \%$ (Kransdorf et al., 1999). In general, LSMFT is an incidental finding, and most patients often complain of longstanding discomfort. The pathogenesis of LSMFT is still under discussion. In general, due to the same point mutation of the GNAS1 gene coding the Gs-alpha protein in both LSMFT and fibrous dysplasia (FD), the lesion is thought to be a variant form of FD (Matsuba et al., 2003) or probably secondary to repeated

(c) The Author(s). 2018 Open Access This article is distributed under the terms of the Creative Commons Attribution 4.0 International License (http://creativecommons.org/licenses/by/4.0/), which permits unrestricted use, distribution, and reproduction in any medium, provided you give appropriate credit to the original author(s) and the source, provide a link to the Creative Commons license, and indicate if changes were made. 
micro-trauma within preexisting fibro-osseous lesions (Heim-Hall \& Williams, 2004). Kransdorf et al. described LSMFT as a result of involutional and ischemic alterations of intraosseous lipogenic lesions (Kransdorf et al., 1999) and others have described LSMFT to be a genuine tumor entity (Ragsdale, 1993).

\section{Case presentation}

A 69-year old male patient was referred for bone scintigraphy for further evaluation of an indistinct ovoid-shaped bone lesion within the left iliac bone. The patient complained of pain in the pelvic region of variable intensity for 8 weeks without any specific cause. There was no history of recent or previous trauma or malignant disease.

High-resolution CT images showed a subchondral lesion of $4 \times 4 \times 2 \mathrm{~cm}$ within the left iliac bone close to the sacroiliac joint with a "landscape" like structure and peripheral sclerosis (Fig. 1, a and b). There was some reduction in sacroiliac joint space without any signs of destruction or invasion by a tumor. On the T2-weighted MRI images, the lesion showed central hypointensity with a hyperintense margin and slight enhancement on the post contrast injection sequences (Fig. 1, c and d).

A three-phase bone scan was performed following intravenous injection of $670 \mathrm{MBq}$ of ${ }^{99} \mathrm{mTc}$-hydroxy-methylene-diphosphonate (HDP). The early vascular phase was unremarkable with no evidence of hyperfusion at this site. On the blood pool images, there was some minor increased activity seen on to the left iliac bone (Fig. 2, a). On the delayed SPECT images, moderately increased tracer uptake was congruent with lesion seen on the CT scan. SPECT/CT images confirmed a "landscape" like lesion (in-homogeneous structure with an enhanced, peripherally rim of increased tracer uptake) (Fig. 2, b, c). Further, two small lesions with a similar pattern ( 3 and $8 \mathrm{~mm}$ in diameter) were seen within the right iliac bone (images not shown).

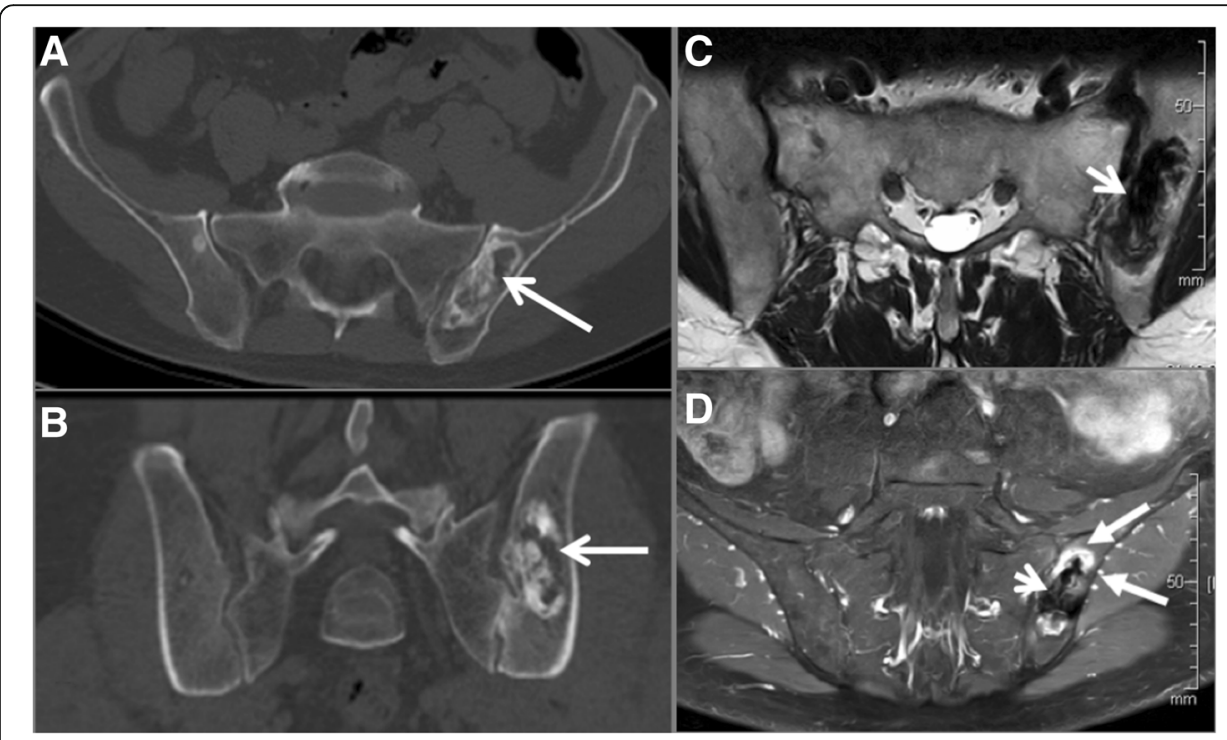

Fig. 1 CT images in transversal (a) and coronal plane (b) showed the "landscape-like" structure of the lesion in the left iliac bone with components of fat density (arrows). MRI revealed a hypointense center (short arrows) of the lesion in both T2 (c) and T1-weighted (d) coronal MRI images with rim enhancement (long arrows) after application of contrast media (d) 


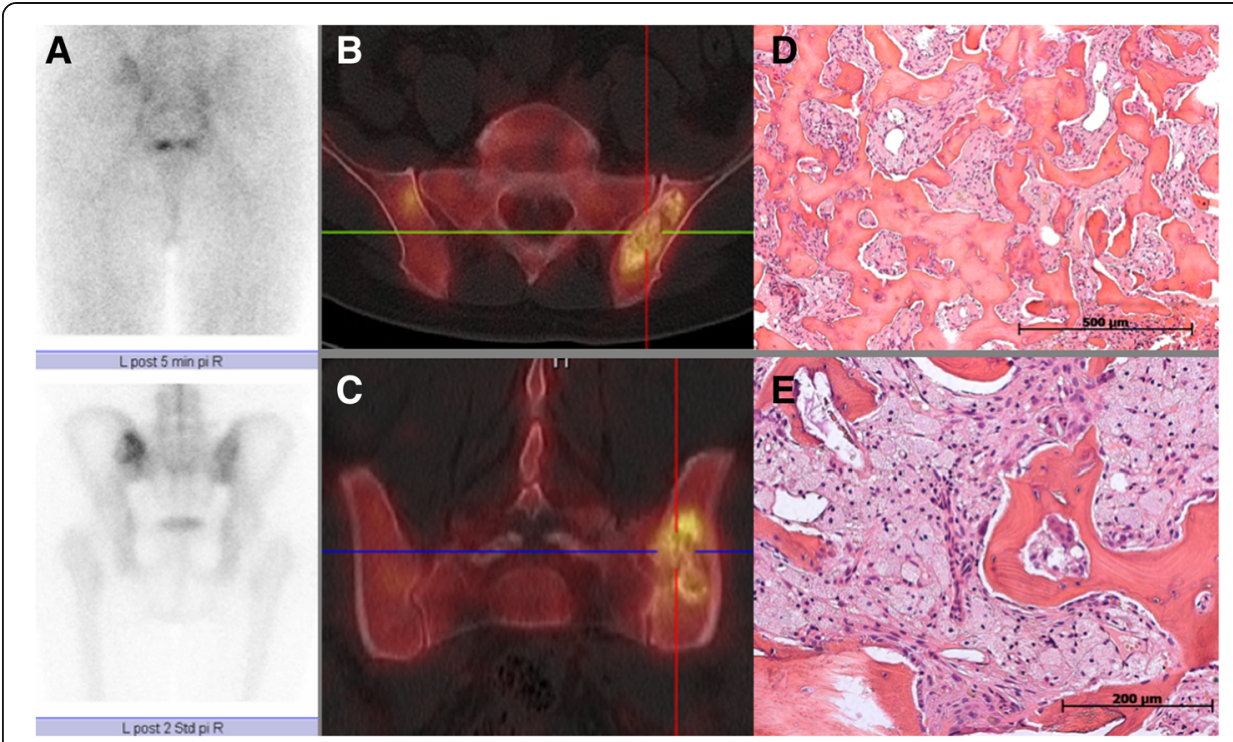

Fig. 2 Planar bone scan (a, dorsal view) showed no hyperperfusion (not shown) but increased blood pool in the left iliac bone in the early images $5 \mathrm{~min}$ p.i. (above) and a moderately enhanced tracer uptake in the delayed phase images (bottom). In SPECT/CT images $2 \mathrm{~h}$ p.i. (transversal view in $\mathbf{b}$, coronal view in $\mathbf{c}$ ), the lesion is easily visible with an inhomogeneous tracer uptake, slightly accentuated in the peripheral zone. Histology (HE staining) revealed lamellar and woven bone with curvilinear and pseudopagetoid features, accompanied by a moderately cellular intertrabecular fibrous stroma (d, magnification 100x). In some areas, clusters of foamy histiocytes were present (e, magnification 200x)

Based on (a) morphological appearance (sharp margins, no destruction of cortical bone, no periosteal reaction, and partly fat containing matrix), (b) bone scan findings (no abnormal hyperperfusion and moderately increased tracer uptake) and (c) vague clinical symptoms, a provisional diagnosis of benign tumor of chondral or fibrous origin with partly myxoid appearance was made.

A CT-guided biopsy was performed due to the persisting symptom (pain) and equivocal imaging diagnosis. The histopathology confirmed a "liposclerosing myxofibrous tumor" (Fig. 2, d, e).

\section{Discussion}

Liposclerosing myxofibrous tumors (LSMFT) are rare, benign fibro-osseous lesions commonly occurring in the femoral intertrochanteric region. It can also be seen occasionally at other sites such as humerus, rib, iliac bone (Kransdorf et al., 1999; Ragsdale, 1993), tibia (Choi et al., 2005; Regado et al., 2016) and skull (Ploof et al., 2018). The reason for this strong predilection is unknown. LSMFT commonly occurs in the 4th decade and affects both men and women equally (Regado et al., 2016). The most frequent presenting symptoms include mild bone pain or occasionally pathological fracture (Dattilo \& McCarthy, 2012).

The diagnosis is often made by conventional radiological imaging such as CT and/or MRI. In general, due to its benign appearance, a biopsy is not performed in the majority of patients (Kransdorf et al., 1999).

Once differential diagnoses such as mixed phenotype metastases, aneurysmal bone cysts, haemangioma, Paget's disease or intraosseous lipoma are excluded on multimodality imaging criteria, the imaging specialist must raise a possibility of LSMFT based on 
Table 1 Indications of bone SPECT/CT in patients suspected with LSMFT

Incidental finding on a bone scan

Atypical solitary bone lesions in patients with history of malignancy

Atypical, incomplete, aggressive, or size (> $4 \mathrm{~cm}$ ) features of LSMFT on X-ray, CT, or MRI Suspicion

of complications related to LSMFT such as pain, fracture, and malignant transformation

the imaging features. In the scenario of pelvic bone localization, imaging specialist must assess according to the anatomical physeal of flat bone equivalents of physeal segments in tubular bones (Nixon, 1976).

If pain or even a pathological fracture is a predominant clinical symptom in a patient with LSMFT, curettage of the lesion and bone grafting of the defect is the treatment of choice and is often associated with a good prognosis in most cases (Deel \& Hassell, 2016). If surgical treatment is not necessary for asymptomatic patients, follow-up examinations are indicated due to the occasional risk of malignant transformation in approximately 10\% (Kransdorf et al., 1999).

Nuclear medicine imaging is rarely required in patients with suspected LSMFT. In general, the tumor is often seen as an incidental finding on a bone SPECT/CT (Table 1) or positron emission tomography/computed tomography (PET/CT) examination. A high ${ }^{18} \mathrm{~F}$-Fluordeoxyglucose (FDG) uptake in LSMFT is reported in a patient with Hodgkin's lymphoma which led to an erroneous upstaging as suspicious bone marrow involvement (Kim et al., 2015). However, after effective chemotherapy due to other lymphoma manifestations, the FDG-uptake in the proximal femur remained unaltered, and the final diagnosis of an LSMFT was confirmed by biopsy.

To the best of our knowledge, this is the first case in the literature using the bone scan with SPECT/CT in a patient with LSMFT. CT and MRI images could not confirm or exclude a malignant process. The size of the lesion, pain, peripheral enhancement post contrast media and probably the unusual localization might account for the uncertainty in the categorization a lesion as benign or malignant.

\section{Conclusions}

Bone SPECT/CT might (a) help in classifying bone lesions by combining metabolic (SPECT) with morphological features (CT) and (b) help in differentiating benign from malignant tumors.

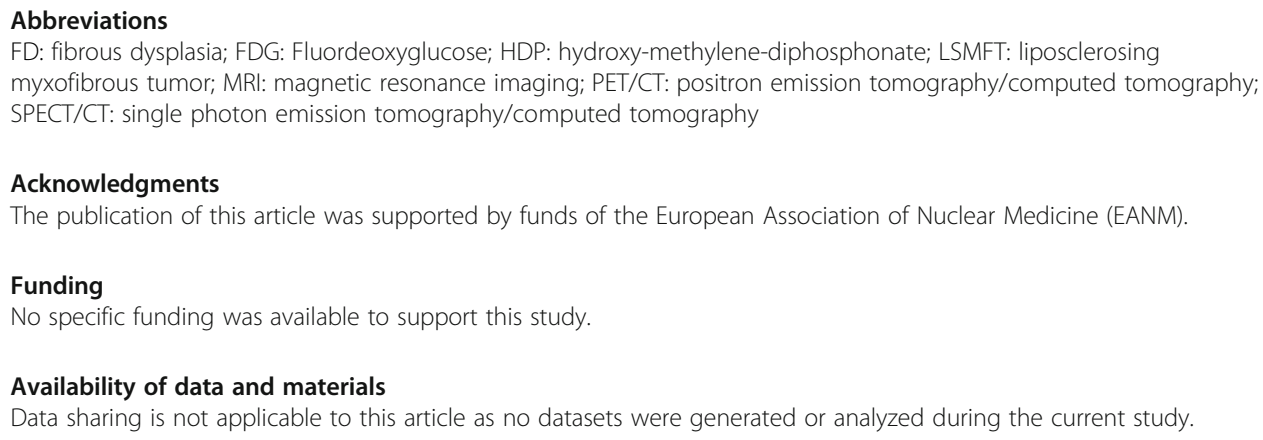

Conception and design: WUK, LA, KS, FP. Acquisition of data, analysis, and interpretation of data: WUK, AML, GS, FP. Drafting the article and final approval of the revised manuscript: WUK, LA, KS, AML, FP. All authors read and approved the final manuscript. 
Ethics approval and consent to participate

For this case report, informed written consent for publication was obtained from the patient. None ethics approval was needed.

\title{
Competing interests
}

The authors declare that they have no competing interests.

\section{Publisher's Note}

Springer Nature remains neutral with regard to jurisdictional claims in published maps and institutional affiliations.

\begin{abstract}
Author details
${ }^{1}$ Nuclear Medicine Spitalerhof, Spitalerstraße 8, Hamburg, Germany. ${ }^{2}$ Department of Nuclear Medicine, Humanitas Clinical and Research Center, Rozzano, Milan, Italy. ${ }^{3}$ Department of Pathology, University Medical Center

Hamburg-Eppendorf, Hamburg, Germany. ${ }^{4}$ Department of Radiology and Nuclear Medicine, Lucerne Cantonal Hospital, Lucerne, Switzerland. ${ }^{5}$ Department of Nuclear Medicine, University Medical Center Lariboisière, Paris, France.
\end{abstract}

Received: 14 September 2018 Accepted: 5 December 2018

Published online: 17 December 2018

\section{References}

Choi LW, Lee YS, Lee JH et al (2005) Liposclerosing myxofibrous tumor in tibia - a case report and review of the literature. Kor J Pathol 39:207-210

Dattilo J, McCarthy EF (2012) Liposclerosing myxofibrous tumor (LSMFT), a study of 33 patients: should it be a distinct entity? lowa Orthop J 32:35-39

Deel C, Hassell L (2016) Liposclerosing myxofibrous tumor - a review. Arch Pathol Lab Med 140:473-476

Heim-Hall JM, Williams RP (2004) Liposclerosing myxofibrous tumor: a traumatized variant of fibrous dysplasia? Report of four cases and review of the literature. Histopathology 45(4):369-376

Kim J, Chen W, Resnik C et al (2015) FDG uptake in liposclerosing myxofibrous tumor causes upstaging of Hodgkin lymphoma. Clin Nucl Med 40(4):325-327

Kransdorf MJ, Murphey MD, Sweet DE (1999) Liposclerosing myxofibrous tumor: a radiologic-pathologic distinct fibro-osseous lesion of bone with a marked predilection for the intertrochanteric region of the femur. Radiology 212:693-698

Matsuba A, Ogose A, Tokunaga K et al (2003) Activating Gs alpha mutation at the Arg201 codon in liposclerosing myxofibrous tumor. Hum Pathol 34(11):1204-1209

Nixon GW (1976) Hematogenous osteomyelitis of metaphyseal-equivalent locations. Am J Roentgenol 130(1):123-129

Ploof J, Shaikh H, Melli J et al (2018) Liposclerosing myxofibrous tumor of the cranial vault: a case report. Neurosurgery. https://doi.org/10.1093/neuros/nyy071 [Epub ahead of print]

Ragsdale BD (1993) Polymorphic fibro-osseous lesions of bone: an almost site-specific diagnostic problem of the proximal femur. Hum Pathol 24:505-512

Ragsdale BD, Sweet DE. Bone. In: Henson DE, Albores-Saavedra J, eds. The pathology of incipient neoplasia. Saunders, Philadelphia. 1986: 381-423

Regado ER, Garcia PBL, Caruso AC et al (2016) Liposclerosing myxofibrous tumor: a series of 9 cases and review of the literature. J Orthop 13:136-139

\section{Submit your manuscript to a SpringerOpen ${ }^{\circ}$ journal and benefit from:}

- Convenient online submission

- Rigorous peer review

- Open access: articles freely available online

- High visibility within the field

- Retaining the copyright to your article 